\title{
De la séquestration des mamans insolvables et leurs enfants dans les maternités des établissements de santé de Lubumbashi : Cas de l'hôpital général Jason Sendwe
}

\author{
Pascal Kakudji Yumba*
}

\begin{abstract}
Our study deals with the carrying out of the medical contract in the maternity of the Jason Sendwe General Hospital in Lubumbashi. The enforcement of the medical contract conveys a notable discrepancy between what is in reality and what should come into force in the maternities of Lubumbashi medical institutions, particularly in the maternity of the above mentioned general hospital. This accounts for the existence of a set of prohibited and questionable practices, namely the sequestration of the mothers and their babies, the over invoicing, and the tip phenomenon that does not take into account the jurisdictional control. The sequestration of the insolvent mothers and their babies reminds one of the ancient Roman institution manus iniectio. This practice is prohibited and in contradiction to the principles of the law. The fore-mentioned sequestration disparages the human person who, like a pledge, warrants the fulfillment of the obligation. There is ground for concluding that the hospital is doing justice to itself in the place of competent jurisdictions entitled to settle such cases of legal dispute.
\end{abstract}

\section{Introduction}

La pratique de la séquestration des patients insolvables dans les maternités les établissements de santé de Lubumbashi s'est imposée comme une sûreté. Cette pratique traduit le souci de sécurité qu'éprouvent les établissements de santé à travers les maternités qui reçoivent les mamans et leurs enfants qui naissent. Cependant, cette pratique est une voie de fait parce qu'elle est une manifestation de la justice privée qui, du reste est prohibée. Aussi, la pratique précitée est une infraction à la loi pénale et une violation des droits de l'homme.

C'est pourquoi, la recherche d'une garantie respectueuse des droits de l'homme et de la dignité humaine s'impose puisque la sécurité juridique se recherche également dans la conclusion et exécution du contrat médical. De tous les cas, le contrat médical est tiraillé entre les faits et les normes dans sa formation comme dans son exécution. Ce qui est paradoxale c'est de constater que, les normes sont menacées de perdre le contact avec la réalité sociale.

* Il est professeur associé à l'Université de Lubumbashi et Doyen de la Faculté de Droit à l'Université Baptiste du Congo. Aussi, il est consultant au Cabinet Yav et associés (www.yavassociates.com; Email : pascalkakudjiyumba@gmail.com). 
Le fait a une emprise considérable sur le normatif au point de nous demander si le droit joue t-il son rôle stabilisateur dans la formation et l'exécution du contrat médical.

Notre étude prend en compte les normes entre elles-mêmes et les rapports qu'elles entretiennent, mais aussi l'espace (la maternité de l'hôpital général Jason Sendwe ${ }^{1}$ ) dans laquelle se déploie le droit et surtout ce qui apparaît publiquement et conflictuelle. La tension entre la factualité et la validité dans la formation, l'exécution du contrat médical traduit une distanciation notable entre ce qui est et ce qui doit être en vigueur dans les maternités des établissements de Lubumbashi particulièrement, la maternité de l'hôpital général cité ci -haut. Ce qu'explique l'existence d'une somme de pratiques prohibées et douteuses (la séquestration, surfacturation, phénomène merci $^{2}$ ) que n'attient pas le contrôle juridictionnel. C'est écart entre fait et la norme pose énormément problème dans la formation et l'exécution du contrat médical.

\section{Objectifs}

- Déterminer les caractéristiques de la séquestration des mamans et leurs enfants dans la maternité de l'hôpital général Jason Sendwe;

- Identifier les causes de séquestration de notre population cible.

\section{Position du problème}

Il est vrai que, le traitement de certains états pathologiques nécessite une hospitalisation de ceux qui en souffrent, c'est le cas de mamans qui se rendent à la maternité pour a couchée leurs enfants. Dans cette occurrence, le contrat médical qui lie les mamans (qui accouchent à la maternité) à l'hôpital général Jason Sendwe est double : hospitalisation et soin de santé de qualité. Ainsi, hospitalisation de mamans et leurs enfants à la maternité est soumis à la réglementation de l'établissement de santé. Cependant, cette soumission n'emporte pas l'aliénation des droits fondamentaux pour les mamans et leurs enfants hospitalisés. Au demeurant, le contrat médical est résiliable par la seule volonté des parties et plus facilement pour les mamans considérées comme patientes. Lorsqu'elles veulent quitter l'établissement

1 Il a été inauguré en 1925, sous l'appellation Hôpital général Prince Léopold dit hôpital « indigène ». Il desservait à ses débuts uniquement les populations noires. Il était exclusivement un hôpital de l'état. Jusqu'au cours des années 1970 hôpital général Jason Sendwe connut une direction partagée entre l'Etat et l'UOC, aujourd'hui Université de Lubumbashi. En 1974, la direction fut confiée à la Général des Carrières et Mines (Gécamines). En 2005 à 2010 la gestion a été de nouveau confiée à l'Université de Lubumbashi et aujourd'hui, c'est l'Etat Congolais qui a la direction. Hôpital général Jason Sendwe à une capacité de plus ou moins 1.200 lits.

2 Kakudji Yumba, Pascal, Les droits des patients et leurs applications dans les établissements de santé de Lubumbashi, Thèse de doctorat, Université de Lubumbashi, Juillet 2014. Ce terme signifie que les praticiens professionnels perçoivent de l'argent supplémentaire auprès des patients qu'ils soignent en dehors de leurs honoraires. 
de santé au cours de traitement, doivent payer au préalable les prix du jour ${ }^{3}$ occasionnés par l'intervention thérapeutique subi, soient mettent en gage les biens meubles pour négocier leur liberté d'aller et de venir afin de garantir l'excuser de l'obligation contracter. A l'inverse, les mamans peuvent suivre le traitement médical et soumise à une hospitalisation pour l'accouchement jusqu'à ce que son état pathologique ne nécessite plus l'hospitalisation. Dans cette occurrence le médecin traitant est tenu de leurs délivrer le bon de sortie et les mamans doivent exécuter son obligation de payer le prix du jour ${ }^{4}$. Mais, il arrive à ce que les mamans soient dans l'impossibilité de payer et curieusement le bon de sortie ne leurs sont pas délivré. Ceci est à dire que, l'établissement de santé séquestrent les mamans insolvables jusqu'au payement complet du prix du jour. Cette pratique est devenue courante dans les établissements de santé de Lubumbashi et particulièrement à l'hôpital général Jason Sendwe.

\section{La recherche de la terminologie}

La pratique à laquelle recours l'hôpital général Jason Sendwe pour garantir l'exécution de l'obligation contractée par les mamans, la précision terminologique s'impose d'autant plus nous ne sommes pas du même avis avec ceux qui qualifient cette pratique de " prise en otage et rétention »

\section{La prise en otage}

Une certaine opinion qualifie de prise en otage, le fait pour un établissement de santé de garder les mamans insolvables et leurs enfants à la maternité de l'hôpital en vue de contraindre celui-ci à payer. Cette vision de chose est malaisément admissible en ce sens qu'elle ne rend pas compte le fait étudié. En effet, l'otage est une personne dont on s'empare et qu'on utilise comme moyen de pression sur quelqu'un. Pourtant, l'hôpital général Jason Sendwe ne s'empare pas des mamans insolvables et leurs enfants à la maternité. Ces derniers se trouvent sous emprise pour effet du contrat d'hospitalisation, l'accessoire du contrat médical. La prise en otage s'analyse comme une « détention d'une personne dans le but de préparer ou faciliter la commission d'un crime, favoriser la fuite ou assurer l'impunité des auteurs ou des complices ou de répondre de l'exécution d'un ordre ou d'une détention $\gg{ }^{5}$ En droit traditionnel, l'otage est une personne remise à un clan pour garantir

3 Le prix du jour est l'ensemble de fais payés par les patients à l'établissement de santé. C'est le cas : honoraires du médecin, hospitalisation, restauration si possible, etc.

4 D'après nos investigations, le bon de sortie est délivré par le gestionnaire ou par l'infirmier en chef de bloc. C'est l'une de ces personnes qui autorise la sortie des mamans et leurs enfants à la maternité.

5 Guillien, R. et Vincent, J., Lexique des termes juridiques, éd., Dalloz, Paris, 2011, p. 349. 
l'exécution d'une obligation. L'individu remis en cautionnement n'est pas réduit en esclavage $^{6}$. Le terme prise en otage et inapproprié pour rendre compte le fait étudié.

\section{La rétention}

Le droit de rétention est un droit du créancier de différer jusqu'au paiement de la restitution d'un bien, qui est la propriété du débiteur et qu'il détient, dans la mesure où existe un lien entre le bien ou sa détention et la créance. ${ }^{7}$ Le droit de la rétention n'existe que sur les choses et non sur les personnes. Cependant, en ce qui concerne les personnes humaines on parle de la séquestration. Ce terme convient et rend compte du fait étudié.

\section{La séquestration}

La pratique de la séquestration ressemble à l'institution antique Romaine, la manus iniec$t i o^{8}$. Le débiteur était lié au créancier corps et bien. De la sorte, le débiteur pouvait, à l'échéance, s'emparer de son débiteur insolvable et le séquestrer jusqu'au complet payement de sa dette ou réputée payer par un membre de sa famille afin de réduire son esclavage. Cette même pratique prohibée qui est appliquée à l'hôpital général Jason Sendwe.

\section{Les situations de séquestration des patients insolvables dans quelques pays africains}

Le patient a pendant longtemps été perçu comme celui dont la santé était altérée, un être faible qu'il fallait protéger. La relation médecin-patient, de type paternaliste, était alors fondée sur le principe de bienfaisance à l'égard de la personne en état de faiblesse. Mais ce type de relation ayant pour fondement la rencontre « d'une confiance et d'une conscience » a montré un certain nombre de limites. Elle a conduit parfois à une dépersonnalisation et à un émiettement de la pratique médicale, et a donné le sentiment que l'on s'intéressait plus à la maladie qu'au malade, à tel organe plutôt qu'à la personne au point que le colloque singulier est parfois apparu comme ayant perdu sa signification humaniste ${ }^{9}$. C'est le cas de la séquestration des mamans insolvables et leurs enfants à la maternité de l'hôpital général Jason Sendwe.

6 Matadi Nenga, Gamanda, La question du pouvoir judiciaire en République Démocratique du Congo, contribution à une théorie de réforme, D.I.N., Kinshasa, 2001, p. 24.

7 T'tink, F., Sûreté et principes généraux sur droit de poursuite des créanciers, Larcier, S.a, Précis de la Faculté de Droit d'Université Catholique de Louvain, Bruxelles, 1956, p. 95.

8 C'est une procédure permettant au créancier de séquestrer le débiteur chez lui et de le garder enchainé pendant 60 jours, période pendant laquelle le débiteur ou un tiers pouvait envisager un moyen d'acquitter sa dette.

9 Anne, L., Bertrand, M. et Didier, T., Droit de la santé, PUF, Paris, 2009, p. 293. 
La séquestration des patients insolvables dans les établissements de santé existe et, est régulièrement prouvée dans plusieurs pays africains qui appliquent les prescrits de la conférence de Bamako de 1989, «l'autofinancement des établissements de santé à travers le recouvrement de frais des soins médicaux auprès des patients qu'ils soignent $»{ }^{10} \mathrm{C}$ 'est les cas de : Kenya, Ghana, République Démocratique du Congo, Zimbabwe, etc.

Au Kenya, les patients riches souscrivent une assurance santé, par la suite bénéficient de soins de santé de qualité. Cependant la majorité de Kenya ne peuvent pas souscrire cette assurance. Ils sont référés ou relégués dans les établissements de santé de deuxième catégorie, où ils sont où ils doivent payer et finissent à être séquestrés lorsqu'ils sont insolvables. Sur ce, Kenya est entrain d'introduire une loi portant protection des mamans pauvres, vulnérables et leurs enfants à la maternité, cependant, cela ne couvrira pas le coût élevé de la chirurgie, comme pour la césariennes. ${ }^{11}$ De même au Ghana, les mamans qui accouchent sont fréquemment gardées à hôpital jusqu'au complet paiement des frais d'accouchement.

En République Démocratique du Congo, une organisation locale de défense des droits de l'homme s'est concentrée sur la situation des jeunes mères à l'hôpital lors de la journée internationale des femmes en 2006, montrant à la fois que la proportion croissante des séquestrations de femmes dans les hôpitaux et leurs rejet par les membres de leurs familles qui ne veulent pas être obligés de payer pour elles. Parmi les femmes séquestrées, certaines sont victimes des viols lorsqu'ils sont complètement isolées et dans l'incapacité de trouver qui paiera pour elle.

$\mathrm{Au}$ Zimbabwe, la séquestration des patients n'est pas une règle, car au moins 28 mères et leurs nouveaux nés ont été séquestrés à l'hôpital d'Harare. Les frais de maternité venaient juste d'augmenter et ces femmes étaient incapables de régler leur frais d'accouchement. $^{12}$

\section{La séquestration des mamans insolvables et leurs enfants à la justice privée}

Le principe universel admet que, nul ne peut se rendre justice à lui-même. Qui conque le ferait, use des voies de fait. Ce qui veut dire que, des moyens non autorisés par la loi pour se rendre justice à lui-même s'exposent à la rigueur de la loi. La justice privée était tolérée dans la société primitive et est prohibée dans la société moderne où elle est autorisée que dans la limite de loi. ${ }^{13}$

Parce que la justice privée est, par principe prohibée, quiconque s'estime lésé dans ses droits dispose d'un recours devant les juridictions de l'Etat, qui ont pour mission de dire le droit contesté entre parties.

10 OMS et UNICEF, Initiative de la conférence de Bamako, Rapport, Mali, 1989.

11 Human Rights Watch, Contexte : Santé publique et pauvreté sur fond de conflit, Rapport, 2010.

12 Ibid.

13 Dans le cas de l'arbitrage et de la compensation. 
Cependant, dans le domaine de droit médical, il ressort qu'à Lubumbashi, il y'a une faible proportion du nombre de jugements déjà rendus au tribunal de paix de Lubumbashi / Kamalondo : de 1990 à 2013 on compte seulement cinq jugements rendus sous la responsabilité civile et pénale du médecin. ${ }^{14}$ En effet, il y a eu: un jugement contre la polyclinique le Jourdain, ${ }^{15}$ la polyclinique Afia, ${ }^{16}$ les cliniques universitaires, ${ }^{17}$ la polyclinique des oliviers, ${ }^{18}$ le centre médical Jemima. ${ }^{19}$ Comparativement au nombre de doléances élevées des patients et de leurs familles, il devrait y avoir plus de jugements sous la responsabilité du médecin au lieu de cinq enregistrés. ${ }^{20}$ Est-ce pour le fatalisme des peuples Africains, particulièrement ceux de Lubumbashi qui ne veulent pas non seulement recourir aux juridictions compétentes de l'Etat pour que la justice soit faite, mais aussi à rechercher les causes du décès parce qu'il a été ensorcelé par une tante paternel ou maternel. Ceci est contraire à la philosophie bantoue de père Pempels : chaque mort doit avoir une cause.

En République Démocratique du Congo, les manifestations de la justice privée s'étalent largement et débouchent à des règlements de conflits qui échappent totalement les instances officielles. Ce type de justice dont la cause principale est le sentiment et la conscience de l'impuissance du pouvoir publique. ${ }^{21}$

14 Jugement rendu par le tribunal de paix de Lubumbashi / Kamalondo, le 12 /04 / 1999. R.P.A 2004 / 2405; jugement rendu par le tribunal de paix Lubumbashi / Kamalondo, le 03 juillet 2003, R.P. 1983; jugement rendu par le tribunal de paix Lubumbashi / Kamalondo, le 31juin 2004, R.P.A 28 62; jugement rendu par le tribunal de paix de Lubumashi / Kamalondo, le 27 mai 2010. R.P. 5155; jugement rendu par le tribunal de paix de Lubumashi / Kamalondo, le 11 mars 2011. R.PA 3898.

15 Jugement rendu le 10 août 1999, R.P.A 2004 / 2405, en cause le Ministère public et la partie civile : monsieur Djita Ntambwe et carole Ngyesu contre le prévenu Tshiza Katenga, docteur à la polyclinique le Jourdain de Lubumbashi.

16 Jugement rendu le 03 juillet 2003, R.P.1983 /. I, en cause le Ministère public et partie civile, Kasonga $\mathrm{M}$ akuta contre le prévenu Wakunga Waratch médecin-chirurgien à la polyclinique Don Bosco de Lubumbashi.

17 Jugement rendu le 31juin 2004, R.P.A 28 62, en cause Ministère public et partie civile, Yves Yumba et Misangambu contre le prévenus Dr. Balloy et Musonda. Civilement responsable : clinique Universitaire de Lubumbashi.

18 Jugement rendu le 11 mars 2011, R.P.A, 3898 en cause le Ministère public et la partie civile : monsieur Vazatis contre le prévenu Kapya Mukeya, Dr. à la polyclinique des oliviers de Lubumbashi.

19 Jugement rendu le 27 mai 2010, RP et le 23janvier 2013, R.P.A, 4163 en cause le Ministère public et la partie civile : monsieur Mukeba Kafuka André contre le prévenu Monsieur Kabulo Lakonga André, Dr. au centre Médical Jemima de Lubumbashi.

20 Kakudji Yumba, Pascal, op.cit., p. 4.

21 Akele, Adau, Le droit de mort, vive le droit! In Congo- Afrique, XXXIX ème année, 1999, n³31. 


\section{Portée de la séquestration des mamans et leurs enfants dans la maternité de l'hôpital général Jason Sendwe}

La séquestration des mamans et leurs enfants dans la maternité de l'hôpital général Jason Sendwe rappelle une institution qu'avait connue le droit primitif : l'exécution des obligations sur la personne qui se résume à une manifestation de la justice privée.

L'obligation est une nécessité juridique par l'effet duquel, le débiteur est astreint envers le créancier à une prestation déterminée. ${ }^{22}$ Dans la Rome antique le débiteur était lié au créancier corps et bien. De la sorte, le débiteur pouvait, à l'échéance, s'emparer de son débiteur insolvable et le séquestrer jusqu'au complet payement de sa dette ou réputée payer par le membre de sa famille afin de réduire son esclavage. C'est ainsi que, Rome a connu la pratique de la «manus iniectio ${ }^{23} »$. La manus iniectio était une procédure permettant au créancier de séquestrer le débiteur chez lui et de le garder enchainé pendant 60 jours, période pendant laquelle le débiteur ou un tiers pouvait envisager un moyen d'acquitter sa dette. ${ }^{24}$ C'est la raison de cet acharnement sur le corps du débiteur, car le créancier ne pouvait s'en pendre à rien d'autre. En effet, les biens n'étaient pas saisissables, ils appartenaient à la tribu et au seigneur. ${ }^{25}$

Dans le droit moderne, il y'a une transformation dans la conception de l'obligation : si celle-ci est moins un lien entre deux personnes qu'un rapport deux patrimoines...il est logique qu'elle s'exécute exclusivement sur les biens. ${ }^{26}$ Ceci veut dire que, l'obligation n'entraine pas un assujettissement corporel du débiteur au créancier, le débiteur est quitte d'argent et s'il est insolvable, l'obligation tend à se dissoudre en liberté. A l'origine cette règle excluant l'assujettissement du débiteur au créancier, le respect de la personne humaine et l'intangibilité du corps. Curieusement la pratique de la séquestration des mamans insolvables et leurs enfants dans la maternité de l'hôpital général Jason Sendwe ressemble à l'institution Romaine, la manus iniectio. A une seule différence est que, le débiteur n'est pas enchainé. Il s'agit d'une pratique qui énerve les principes de Droit moderne. La pratique précitée, réifie la personne humaine qui, comme le gage garantit l'exécution de l'obligation. Ce qui est inconcevable pour l'humanité et les établissements de santé d'admettre et de tolérer que les mamans insolvable et leurs enfants puisse aliéner sa personne.

Bien plus, cet acharnement sur le corps de mamans et leurs enfants sont un mépris du principe du gage commun des créanciers consacré par l'article 243 de loi n $73 / 021$ du 20 juillet 19973 qui exclut l'exécution des obligations sur la personne. En effet, rien n'autorise les établissements de santé de séquestrer les mamans insolvables et leurs enfants sans au

22 Kalango, Mbikayi, Responsabilité civile et socialisation des risques, $2^{\text {ème }}$ éd., PUZ, Kinshasa, 1979, p. $152 .$.

23 Michaud, G., Hacquard, G., Dautrey, J. et Maisani, O., Guide romain antique, éd., Hachette, Paris, 1952, p. 82.

24 Deslauriers, J., Précis de droit des sûretés, Wa. Ltd, Montréal. 1990, p. 15.

25 Kalongo, Mbikayi, op. cit., p. 15.

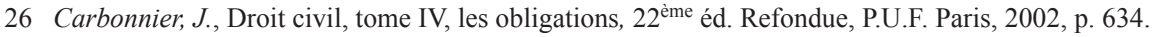


préalable obtenir leurs consentements libre et éclairé. Au demeurant, le corps humain ne doit pas être considéré comme une sûreté parce qu'il partage la destination de la personne dont il fait partie intégrante comme elle a une valeur extrapatrimoniale. Dès lors, l'homme ne peut pas disposer à des fins purement économiques. ${ }^{27}$ Surabondamment, toute convention qui met en jeu la liberté d'un individu est nul d'effet, car l'être humain est hors commerce.

\section{Conséquences juridiques de la séquestration des mamans insolvables et leurs enfants à la maternité de l'hôpital général Jason Sendwe}

La séquestration des mamans insolvables et leurs enfants à la maternité de l'hôpital général Jason Sendwe entraine des conséquences juridiques. Elle est une entrave à la liberté individuelle disposée par la constitution de la République Démocratique du Congo qui dispose en son article 2 alinéa 1 que, « la constitution garantie l'inviolabilité des libertés et des droits fondamentaux ».

La constitution est un reflet d'une conception du rôle de l'Etat dans la société. En outre, elle est une certaine philosophie politique. La jouissance effective des droits fondamentaux reconnus à tous est proclamée par la constitution et ne peut assurée pleinement et efficacement qu'avec l'appui du droit répressif. ${ }^{28}$ Sur ce, l'Etat fait de l'homme la finalité du droit et les pouvoirs publics sont tenu de sécuriser la personne humains. Ainsi, le législateur congolais a érigé en infraction l'entrave à la liberté individuelle, valeur fondamentale qui se situe au carrefour entre la théorie de l'Etat et celle de la justice.

L'article 67 alinéa 1 du Code pénal congolais livre II dispose que, « est puni d'une servitude pénale d'un à cinq ans celui qui, par violences, ruses ou menaces a enlevé ou fait enlever, arrêté ou fait arrêter arbitrairement, détenu ou fait détenir une personne quelconque $»$.

En effet, la question est de savoir : est-ce que la pratique de la séquestration des mamans insolvables et leurs enfants à la maternité de l'hôpital général Jason Sendwe tombe-telle sous le coup de l'article sus-évoqué?

\section{La recherche des causes de la séquestration des mamans insolvables à la maternité}

Il n'existe pas d'effet sans cause. Sur ce fait, la pratique de la séquestration est un effet qui demande de rechercher les causes. Plusieurs causes peuvent expliquer la pratique de la séquestration de mamans insolvables et leurs enfants à la maternité de l'hôpital général Jason Sendwe. Nous pensons que, trois d'entre elles sont significatives et méritent d'être

27 Dierkens, R., Les droits sur corps, une mise à l'épreuve du droit subjectif, éd. Fernand Larcier, Bruxelles, 1966, p. 30.

28 Luzolo, Nkakala et Ali, La pauvreté urbain dans l'Afrique subsaharienne : cas de la ville de Kinshasa, CEPAS, Kinshasa, 1999, p. 25. 
retenus notamment, la crise économique, la délinquance de l'administration publique et l'inefficacité du régime des sûretés dans l'environnement médical.

\section{La crise économique}

Les pays de l'Afrique subsaharienne, parmi lesquels nous trouvons la République Démocratique du Congo, présentent une situation économique à plaindre. Ces pays ont toujours eu un revenu faible et connaissent une stagnation économique due à une forte croissance démographique. Dans cet environnement, le République Démocratique du Congo en dépit de ses potentialités importantes en terme de richesses agricoles et minières, reste un pays pauvre, dont l'indice de développement humain (IDH) du pays est évaluée à 0,431 et donc en déca du seuil de pauvreté fixé à 0,50 (PNU et Banque mondial). Le pouvoir d'achat ne fait que dégringoler et l'accès aux services de santé de qualité devient difficile pour le congolais moyen. Dans cette économie minée, chaque citoyen congolais se débrouille pour survivre donc, c'est le retour au fameux l'article 15 initié par le maréchal Mobutu Sese Seko. ${ }^{29}$

L'économie congolaise a détruit la quasi-totalité des entreprises publique, le citoyen congolais est appelé à tout imaginer pour survivre dans cette environnement de précarité. Notre observation est que, l'exercice de la profession médicale est une entreprise rentable contrairement à la disposition de l'article $6 \mathrm{du}$ Code déontologie médicale qui dispose que « l'exercice de la profession médicale ne doit à n'aucun cas ni d'aucune manière être placée dans le commerce ». Eu égard de ce qui précède, la pauvreté est généralisée et que la maladie attrape qui elle choisit, la conséquence est que le contrat conclu dans cette environnement peut être que pécuniaire et malaisément supportable pour les mamans économiquement faibles hospitalisées dans la maternité de l'hôpital général Jason Sendwe. Le problème de la pauvreté qui rend difficile l'accès aux soins de santé de qualité n'est pas seulement régulier l'apanage de la République Démocratique du Congo, mais aussi d'autres pays africains. C'est le cas : du Burundi où l'accès aux soins de santé de qualité a été rendu plus difficile pour les patients démunis depuis l'adoption du système de recouvrement des frais, en févier 2002. Ces derniers, sans qu'ils soient tenus compte de leur moyen, doivent régler eux-mêmes des frais médicaux engagés, y compris les consultations, les analyses, les médicaments, les fournitures et leurs séjour à l'hôpital. ${ }^{30}$

La pratique de la séquestration des mamans insolvable et leurs enfants dans les maternités des établissements de santé de Lubumbashi devient une réalité endémique pour les économiquement faibles. Elle est dans une certaine mesure bénéfique pour les établissements de santé, car les mamans et leurs enfants séquestrés à la maternité sont tenu de payer le prix du jour, outre que les frais occasionnés par l'intervention thérapeutique dont elles

29 Matadi Nenga, Gamanda, op. cit., p. 24.

30 Mit, P. H., Gorik, O., Sally, H. et Andrew, D., A population deprived of basic health care, The British Journal of General Practice, Burundi, Août 2004, pp. 634 et 635. 
souffraient. Puisque le frais officiel d'accouchement normal pour une maman revient à 18.000 FC (dix huit mille Francs congolais) soit 20\$ (vingt dollars américain). Dans 1'informelle, elle doit payer 45.000 FC soit 50\$ pour avoir été soignée et hospitalisée pendant trois jours. Cette pratique également remet en cause la disposition de la circulaire du 20 juin 1982, relative à la fixation de tarif dans les établissements de santé de la République Démocratique du Congo. Dans le même ordre d'idée, il n'y a plus l'application d'égalité dans d'accès aux soins de santé de qualité. ${ }^{31}$

Par ce moyen, les praticiens professionnels trouvent un autre moyen de renflouer la caisse à travers la facturation informelle. ${ }^{32}$ La séquestration des mamans et leurs enfants dans les établissements de santé visent une spéculation mercantile sur hébergement. Pourtant, cet hébergement ne fait pas partie du contrat conclus entre les mamans hospitalisées à la maternité de l'hôpital général Jason Sendwe. Autrement dit, la séquestration des mamans insolvables et leurs enfants dans les maternités constituent un contrat imposé par l'établissement de santé de Lubumbashi. Le quel contrat ne fait que qu'aggraver la situation des mamans séquestrées.

\section{La déliquescence de l'administration publique}

Depuis 1960, la République Démocratique du Congo est caractérisée par une déliquescence continuellement varié partout au long de son parcours. Aujourd'hui, elle s'est désorganisée davantage. C'est pour cette raison que, la population de la République Démocratique du Congo n'est estime qu'approximativement et les résidant d'une commune ne sont connus de celle-ci. L'insécurité qui résulte du dysfonctionnement de l'administration publique est grand, chaque citoyen déménage, voyage comme bon lui semble sans que cala ne soit mentionné dans le registre de bureau de l'Etat civil. De plus, les adresses des citoyens congolais sont inconnues de l'administration publique, si elles sont connues, alors douteuses tellement ils déménagent.

En effet, le droit de créance est un droit personnel. Et, la créance est un lien de droit entre deux personnes. Ce qui justifie que la personne qui est dans les liens d'une obligation contractuelle avec les autres doit être localisable. Il en est de même pour l'établissement de santé, qui a établi la décharge avec les mamans hospitalisées à la maternité afin de revenir quelques semaines après pour honorer leurs factures, ces dernières doivent être localisables par l'administration de l'établissement. Le constat est qu'il difficile d'avoir une adresse sûre et précise, surtout de le retrouver après les soins reçu, car les frais à payer constitues une garantie locative dans un autre quartier de la ville de Lubumbashi. Devant cette insécurité, les établissements de santé de Lubumbashi trouvent la formule équitable de séquestre les mamans insolvables et leurs enfants pour garantir l'exécution de l'obligation contractées

31 Camille, K. et Aurelia, D., Les droits des malades, éd. Dalloz, Paris, 2012, p. 66.

32 Jaffre, J. et Sardano, $O$. de, Une médecine inhospitalière, les difficiles relations entre soignants et soignés dans les cinq capitales d'Afrique de l'Ouest, éd., Karthala, Paris, 2003, p. $256 .$. 
par ces dernières. Parce que, la chance de voir les mamans qui ont reçu les soins de santé à crédit revenir payer est minime.

\section{L'inefficacité du régime des sûretés dans l'environnement des soins}

Le commerce juridique appelle d'idée de garantie, de sûreté. ${ }^{33}$ L'arsenal juridique des sûretés sont régies par la loi $n^{\circ} 73 / 021$ du 20 juillet 1973 telle que subséquemment modifiée. En effet, la naissance des sûretés a suivi au cours de temps une évolution en rapport avec les mentalités et le contexte socio-économique de chaque peuple. ${ }^{34}$ Le législateur congolais s'est approprié d'un droit étranger par le fait de la colonisation, car la loi du 20 juillet 1973 citée ci-haut est une reproduction identique de législateur colonial. Si le problème de sûreté, de garantie est universel, cependant la manière d'organiser celle-ci n'est pas le même dans tous les pays. ${ }^{35}$ Chaque société doit organiser son régime de sûreté suivant la mentalité de sa population et son contexte socio-économique.

Notre régime des sûretés ne suivent pas la réalité sociale afin d'édicter une loi spécifique en la matière. C'est une vérité fondamentale, que si souvent l'on perd de vue : « le droit derrière son aspect technique est avant tout, une vision du monde, une traduction de la conception qu'un peuple se fait de l'organisation social $»{ }^{36}$ Le problème de la séquestration devient dramatique dans la situation économique difficile, laquelle le phénomène de pauvreté est permanent. La maladie touche tout le monde, riche ou pauvre et le besoin de soins de santé de qualité se fait sentir. Dans ce contexte, le pauvre ne savent pas donner de garanties à l'établissement de santé (hôpital général Jason Sendwe) ou si la garantie offerte n'est pas suffisante, les mamans économiquement faible pourra être séquestrées jusqu'au paiement complet de prix du jour. Les sûretés organisées par la loi précitée sont inefficaces dans le domaine de soins de santé. Généralement, les établissements de santé via leurs maternités agissent d'abord, c'est-à-dire dispenser les soins et réclament les prix du jour après. ${ }^{37}$ En effet, exiger des garanties avant le traitement est contraire à l'exercice de la profession médical quoi qu'on demande aux mamans à la maternité une caution pour l'accouchement. ${ }^{38}$ Lorsque celle-ci se relève insuffisante après le traitement subi ou si elle n'a pas été fournie, les mamans et leurs enfants seront séquestrées jusqu'au payement complet par l'établissement de santé. La séquestration des mamans insolvables et leurs enfants relève d'une pratique ou coutume " contrat legem ${ }^{39}$ " car elle se développe au contrairement à la loi.

33 Le terme évoque l'idée de sécurité, de protection, d'assurance, etc.

34 Kalambay, Lumpungu, Droit civil, Volume I, Régime général des biens, PUZ, collection Mafundisho, Mateya, Kinshasa, 1990, p. 192.

35 Matadi Nenga Gamanda, op. cit., p. 27.

36 Akele, Adau, op. cit.

37 En cas d'intervention d'urgence.

38 Cas de la polyclinique Afia et clinique de Général des Carrières et des Mines.

39 Une pratique qui est contraire à la loi. 
La coutume est une source de la loi dans le sens que ce dernière et la loi crée le Droit. Puis qu'il s'agit de deux sources de la règle de Droit. Il est vrai que, le régime des sûretés organise l'exécution des obligations uniquement sur les biens et non sur sa personne. Pourtant, la pratique de la séquestration des mamans et leurs enfants est en conflit avec le régime de sûreté et il est important de déterminer la portée et les conséquences juridiques qu'elle peut entrainer.

\section{$X$. Recherche des solutions pour garantir l'exécution du contrat médical entre les mamans insolvables et l'hôpital général Jason Sendwe}

La pratique de la séquestration des mamans insolvables et leurs enfants à la maternité de l'hôpital général Jason Sendwe est considérée comme une sûreté. Elle traduit le souci de sécurité qu'éprouve l'établissement de santé précité qui reçoivent et soignent les patients (es). Cependant, ladite pratique est une manifestation de la justice privée et prohibée. Elle constitue une violation aux principes des droits de l'homme et à l'article 67 du Code pénal Congolais. La recherche d'une garantie respectueuse trouve son fondement dans la Déclaration Universelle des droits de l'homme en matière de la dignité humaine.

\section{Le pouvoir de contrainte de l'hôpital général Jason Sendwe}

L'hôpital général Jason Sendwe sait astreindre d'exécuter une prestation déterminée, c'est un pouvoir de contrainte contenu dans toute obligation. Le pouvoir de contraindre est là, l'échéance pour rappeler les mamans insolvables qu'elles sont liées corps et biens. La force contraignante dont l'obligation est assortie normalement par les pouvoirs de l'hôpital cité ci-haut vise les biens meubles des mamans insolvables (mis en gage), exceptionnellement, dans les coercitions sur les personnes (les mamans et leurs enfants ${ }^{40}$ ).

Le droit de gage commun de l'hôpital général Jason Sendwe sur les biens des mamans insolvables procède de la liaison entre l'actif et le passif du patrimoine. C'est-à-dire que, l'actif répond du passif. Cette liaison fut faite pour la première fois à Rome par le préteur Retulius qui voulait mettre à néant tout contrat engageant la liberté d'une personne. Il organisa un mode d'exécution sur les biens du débiteur, reforme capital qui avait établit un lien entre actif et le passif du patrimoine. ${ }^{41}$ L'article 245 de la loi $n{ }^{\circ} 73 / 021$ du 20 juillet 19973 portant régime général des biens, régime foncier et immobilier et régime de sûreté consacre le principe de la sujétion uniforme des biens du débiteur à l'accomplissement de ses dettes en disposant que : " tous les biens du débiteur présents et à venir, sont le gage commun de ses créanciers et le prix s'en distribue entre eux par contribution, à moins qu'il n'y ait entre les créanciers des causes légales de préférence ». Il en résulte que, tous les biens des mamans hospitalisées à la maternité de l'hôpital général Jason Sendwe, mobiliers,

40 Carbonnier, J., op. cit., p. 633.

41 Deslauriers, J., op. cit., p. 2. 
corporels sont compris dans le gage commun. Les biens visés sont non seulement les biens présents mais aussi ceux avenir que les mamans pourrons acquérir après l'engagement avec l'établissement de santé cité ci-haut.

Ce principe se comprend si l'on considère que les soins de santé dispensés par le praticien professionnel aux mamans et à leurs enfants sont à crédit lors de leurs engagements. C'est pour cette raison, elles doivent emprunter et s'engager à payer ultérieurement en utilisant les ressources qu'elles auront dans d'avenir. ${ }^{42}$ De la sorte, l'établissement de santé ne doit pas, en vertu du gage commun, refuser de dispenser les soins de santé de qualité aux mamans et à leurs enfants nés vivant et viables qui ne sait pas payer dans l'immédiat le prix du jour. Elles paieront au moyen obtenu des ressources futur. Sans oublier que la dette est quérable et non portable.

Précisons que, le gage commun des créanciers n'est pas un droit réel de gage, c'est-àdire une sûreté. Il faut seulement entendre que, le créancier a sur les biens du débiteur une série de pouvoirs plus énergiques. Le plus essentiel des pouvoirs est celui de saisir les biens : c'est l'exécution forcée, qui donne à la créance son utilité finale. ${ }^{43}$ Tout créance est affectée d'un vice : l'instabilité du débiteur (les mamans insolvables). C'est ainsi que, les forts de leur droit de gage commun des créancier peuvent, sans poursuivre l'exécution forcée, jouer à la prévention et prendre des mesures visant la conservation du patrimoine du débiteur pour conjurer l'insolvabilité de leur débiteur.

\section{Garantie l'exécution de l'obligation des mamans insolvables envers l'hôpital général Jason Sendwe}

Lorsque les mamans font garantir l'exécution de l'obligation par une sûreté, il se protège par là même contre l'insolvabilité de son débiteur. C'est ainsi, la sûreté protège le l'hôpital général Jason Sendwe contre l'insolvabilité des mamans hospitalisées à la maternité à défaut de paiement à l'échéance. Le titulaire d'une créance réelle échappe à la loi du concours entre créanciers chirographaires. Et s'il dispose d'une sûreté personnelle, le créancier est en droit d'exiger le payement d'un autre débiteur obligé en sens du premier. ${ }^{44}$ Ces sûreté sont gouvernées par quelques principes fondamentaux qui sont une exception à la règle du partage par concurrence entre créanciers (hôpital général Jason Sendwe) des même mamans insolvables (débiteur) prévue par l'article 245 de la loi n73/021 du 20 juillet 1973 portant régime général des biens, régime foncier et immobilier et des sûretés. Cependant, cet article introduit, in fine, une exception : les causes légitimes de préférence entre créanciers. Ainsi, trois principes fondamentaux gouvernent les sûretés réelles : les débiteurs personnels, le gage commun des créanciers ainsi que l'égalité et le concours entre créanciers. Dans le contexte de notre travail, les mamans insolvables ne sont pas considérées comme les débiteurs

42 Ibid.

43 Carbonnier, J., op. cit., p. 637.

44 T'tink, F., op. cit., p. 5. 
personnels engagent, en vertu de l'article 245 sus-évoqué, tous les biens présents et à venir à l'apurement de sa dette. Par contre, comme débiteurs réels qui affectent un de ses biens à la garantie de paiement du prix du jour à l'hôpital général Jason Sendwe. C'est pour cette raison Jacques Deslauriers nous renseigne que « le débiteur réel n'est tenu qu'à l'égard d'une chose affectée au paiement d'une créance. ${ }^{45}$

En définitive, il résulte que les sûretés sont indispensables pour l'obtention du crédit par les mamans insolvables à maternité de l'hôpital général Jason Sendwe. Les sûretés constituent une garantie pour l'exécution de l'obligation surtout lorsque l'on sait qu'à l'échéance, le droit de l'établissement de santé précité est précaire pour recouvrer les frais auprès des mamans hospitalisées à la maternité. C'est-à-dire, il supporte l'insolvabilité des mamans. Au reste, ce qui nous intéresse dans le cadre de notre recherche se sont les sûretés qui peuvent garantie l'exécution de l'obligation contractée par les mamans hébergées à la maternité de l'hôpital général Jason Sendwe. Il s'agit de : du cautionnement, du gage des biens meubles pour négocier leurs libertés d'aller et de venir en cas du non-paiement de prix du jour.

\section{Le cautionnement}

Le « cautionnement ${ }^{46}$ » est une sûreté personnelle qui a été pratiquée dans tous les pays du monde y compris la République Démocratique du Congo à travers l'article 337 de la loi n Le cautionnement est un contrat par lequel une ou plusieurs personnes s'obligent envers les autres (créanciers) à acquitter la dette d'un tiers, dans le cas où le tiers ne l'acquitterait pas lui-même.

73/021 du 20 juillet 1973 sus-évoquée qui dispose que « celui qui se rend caution d'une obligation se soumet envers le créancier à satisfaire cette obligation, si le débiteur n'y satisfait pas lui-même ». Cette définition n'est pas à l'abri des criques. Car elle ne mentionne pas un trait caractéristique du cautionnement : le recours subrogatoire de la caution après le paiement fait par elle. C'est-à-dire faut-il ajouter à cette définition sauf, recours subrogatoire contre celui-ci.

Le cautionnement est soumis, comme tout contrat, aux conditions de validité des conventions prévues à l'article 8 du Code civil congolais livre III, à savoir : le consentement, la capacité, l'objet certain et une cause licite. En plus de ces « caution ${ }^{47}$ » conditions générales, le cautionnement obéit à certaines conditions particulières relatives à la caution, au consentement requis pour se porter caution ainsi qu'aux obligations susceptibles d'être cautionnées.

45 Deslauriers, J., op. cit., p. 7.

46 Le cautionnement est un contrat par lequel une ou plusieurs personnes s'obligent envers les autres (créanciers) à acquitter la dette d'un tiers, dans le cas où le tiers ne l'acquitterait pas lui-même.

47 La caution est une personne qui s'engage à payer au cas où le débiteur principal ne paierait pas. 
a. Conditions relatives à la caution

Le débiteur obligé à fournir une doit en préparer une qui ait la capacité de contracter et qui ait un bien suffisant pour répondre de l'obligation. ${ }^{48} \mathrm{Au}$ terme de la loi, il résulte que la caution fournie doit avoir la capacité de contracter. Cela va de soi d'autant plus que, le cautionnement est un contrat. Il est en exacte de dire que, le cautionnement est un acte dispositif, la caution qui s'oblige à payer dispose de ce qu'elle s'oblige à donner au créancier. En effet, payer c'est disposer. Ainsi, tous les incapables désignés par l'article 215 du Code de la famille congolais ne peuvent pas cautionner. Il est aussi de cas où une personne bien que pleinement capable, n'ait pas la capacité de disposer, l'exemple donné par l'article 499 du Code de la famille. ${ }^{49}$

\section{b. Extinction du cautionnement}

L'obligation de la caution étant accessoire s'éteint en même temps que l'obligation principale qu'elle garantit : paiement, novation, remise de la dette, etc. ${ }^{50} \mathrm{C}$ 'est en ce sens que, l'article 358 de la loi 73/021 du 20 juillet 1973 sus-évoquée dispose que « l'obligation qui résulte du cautionnement s'éteint par les mêmes causes que les autres obligations ". Précisons que, le cautionnement s'éteint également toutes les fois que le créancier viole l'obligation mise à sa charge. C'est-à dire il empêche, par son fait, le recours subrogatoire. ${ }^{51}$ Le cautionnement, sûreté personnelle, a fait ses preuves au cours du temps, cependant, il a perdu son prestige depuis que les sûretés réelles se sont développées.

Le cautionnement a longtemps fonctionné dans la société familiale. De nos jours, avec l'affaiblissement de la solidarité familiale portée par les velléités du modernisme, le cautionnement est devenu moins usité. Surabondamment, le cautionnement semble être une sûreté fragile en ce sens que la caution peut devenir à leur tour insolvable. Nous pensons que, la garantie que procure le cautionnement est précaire et l'insolvabilité de la caution comme celle du débiteur principal (mamans hospitalisée à la maternité) reste suspendu audessus du créancier (Hôpital général Jason Sendwe) comme épée de Damoclès.

Il est aisé de constater que, le cautionnement est une sûreté qui a perdu son prestige, tant la garantie qu'elle confère est fragile. C'est la raison pour laquelle, l'établissement de santé (l'hôpital général Jason Sendwe ou les praticiens professionnels selon le cas ne peuvent pas y recourir. Cependant, lorsque l'état de santé des mamans et leurs enfants à la ma-

48 Article 343 de la loi 73/021 du 20 juillet 1973.

49 «Quel que soit le régime matrimonial et les modalités de la gestion de ce régime, l'accord des deux époux est nécessaire pour faire une donation de plus de 500 zaïres ou cautionner la dette d'un tiers pour un montant supérieurs à 5.000 zaïres, sur les biens communs ou propres de l'autre époux ».

50 Dupoy, C. et Ressayer, M., Précis de droit civil, Tome II, Obligation-Sûretés-Principaux contrats, Donod, Paris, 1980, p. 183.

51 La subrogation est l'opération de substitution d'une personne ou d'une chose, par une autre, dans un rapport juridique. 
ternité nécessitent une hospitalisation, les établissements de santé ont coutume de demandé à ces dernières de payer une somme équivalente à autant de jours d'hospitalisation. Une nuance doit être faite à ce stade. La caution, nous savons, est une personne qui s'engage à payer au cas où le débiteur principal ne paierait. Le terme caution qu'on a coutume d'utilisée pour désigner la somme d'argent que les mamans paient pour leurs hospitalisation est inapproprié. Mieux, on devrait parler de cautionnement parce que ce terme désigne à la fois, l'opération consistant à garantir le créancier (l'établissement de santé) contre l'insolvabilité de son débiteur (les mamans hospitalisées) en lui désignant des débiteurs supplémentaires qui paieraient au cas où les premières ne le feraient pas. C'est le contrat qui donne naissance à cette sûreté personnelle. Le terme cautionnement sert également à désigner une somme d'argent qui personne remet à son contractant en garantie de sa dette. ${ }^{52}$

La pratique sus-évoquée est un paiement anticipatif que l'on appelle par défaut caution. La preuve est que, la caution n'est pas perçue comme garantie au cas où les mamans ne paieraient le prix du jour, mais comme un véritable paiement. De la sorte, lorsque les mamans font plus de jours, elles ne seront tenues que d'ajouter la somme correspondante au nombre de jours excédent ceux couverts par la caution. Si les mamans font moins de jours, elles lui seront remises le montant correspondant à la caution défalquée de prix du jour. Cette pratique qui se généralise n'est pas à l'abri des critiques. Outre la sécurité qu'elle accorde à l'établissement de santé. Elle crée une situation d'exécution pour ceux-là qui ne peuvent pas constituer cette caution. Etant une condition préalable d'intervention thérapeutique. Elle est susceptible de constituer un obstacle à l'accès aux soins de santé de qualité pour les économiquement faibles. Nous sommes en train d'assister impuissamment à la célèbre loi du plus fort et l'intervention des pouvoirs publics s'impose.

\section{Suggestion}

Devant l'inadéquation des garantir le droit commun dans l'exécution du contrat médical, l'établissement de santé se protège par la pratique de la séquestration des patients insolvables jusqu'au complet paiement du prix du jour. En guise de suggestion, nous proposons :

\section{La prohibition de la pratique usitée à l'hôpital général Jason Sendwe}

La pratique de la séquestration des mamans insolvables et leurs enfants est une infraction à la loi pénale et révèle l'existence d'un droit maquisard, car c'est l'exercice d'une justice privée. Comme le mal frappe la majorité des congolais, seule l'intervention du législateur s'avère nécessaire pour en rayer la pratique de la séquestration des patients insolvables en général et en particulière celle des mamans et leurs enfants à la maternité de l'hôpital général Jason Sendwe. Et même la prohibition de la soumission de tout traitement médical 
au payement préalable des frais a été portée par une circulaire administrative du secrétariat général à la santé publique $\mathrm{n}^{\circ} 1251 / 001 / 82$ du 16 octobre 1982.

\section{La création des mutuelles de soins}

La pratique de la séquestration et de la soumission de tout traitement médical au payement préalable des frais de soins n'apportera pas la sécurité exigée. Il faut garantir les droits de toutes les parties, car les soins médicaux ne sont pas gratuits. La maladie est un risque indéniable. Elle est lourde de conséquences pour les économiquement faibles. C'est pour cette raison qu'elles sont séquestrées à la maternité de l'hôpital général Jason Sendwe jusqu'au paiement complet. Sur ce, il faut instaurer le système des sociétés mutuelle des soins. En effet, notre démarche est dictée par le fait que la séquestration des mamans et leurs enfants à la maternité de l'hôpital général Jason Sendwe comptent sur l'intervention des parents, amis et autres connaissances des personnes séquestrées pour acquitter la dette.

Ce qui suppose une certaine solidarité. Cette dernière tend à nos jours à disparaître dans les milieux urbanisés à causes des actes d'égoïsme devenus fréquents dans la vie de l'individu vivant dans ce milieu précité. De plus individu acquiert une mentalité et des habitudes : il aspire par son travail individuel à une économie fondée sur le profit personnel, il découvre aussi la propriété individuelle et veut consolider de nombre des membres comme des parasites. ${ }^{53}$ Devant cette situation, nous pensons qu'il est possible de créer, au moyen des connaissances juridiques, une solidarité artificielle incarnée dans les sociétés mutualistes. Les sociétés mutualistes ont pour mission de procurer à leurs membres, en échange d'une cotisation, certains avantages en vu d'améliorer leur condition morale ou leur situation économique. ${ }^{54} \mathrm{Il}$ s'agira d'une société mutuelle organisée à partir de la base, au niveau de la commune par exemple « un tel système est non seulement concevable, mais réalisable en République Démocratique du Congo... il se tisse toujours un réseau de liens de solidarité qui va de la famille la plus stricte aux relations les plus large comprenant parfois des amis ou de simple connaissances. La pratique de la ristourne, la tontine est déjà l'expression manifeste d'une certaine solidarité. Elle devra être dépouillée de son caractère actuel pour devenir un véritable système de solidarité collective, organisé sous forme de mutuelles. ${ }^{55}$

53 Mukadi, Bony, Quelle sécurité sociale pour la troisième République? Esquisse d'un modèle sur base des décisions et recommandations de la conférence nationale souveraine, éd. Ntobo, Kinshasa, 1993, p. 17.

54 Kalambay, Lumpungu, op. cit., p. 168.

55 Discours prononcé au Palais du peuple par le président Fondateur du MPR, président de la République du Zaïre, le maréchal Mobutu Sese Seko, à l'occasion de la prestation de son serment constitutionnel In Bilan et perspective, Kinshasa, 1984. 
3. La création d'un fonds provincial pour la santé comme fut le cas avec le fonds National Médico-Sanitaire (FONAMES) de 1985

En 1985, la République Démocratique du Congo avait adhéré à la charte de développement sanitaire en Afrique. Aussi, elle avait délimité le pays en zone de santé tout en créant le fonds National Médico - Sanitaire dont l'objectif était de coordonner au nom du Ministère de la Santé, l'aide des partenaires aux zones de santé. Cependant, celui-ci avait échoué de sa mission, laissant son objectif être assuré par les zones de santé.

Sur ce fait, les conditions économique - financières et structurelles ne permettent pas à la population congolaise de se souscrire une assurance maladie obligatoire, qui est un modèle de fonctionnement idéal pour tout pays. Il est possible de tenter l'expérience de créer un fonds de solidarité pour la santé au niveau provincial, lequel constituera un appui financier supplémentaire auprès du secteur de santé en parallèle avec la part du budget national affecté à la santé. Ce fonds pourraient être crée en concertation interministérielle, en instituant par exemple une taxe sur l'un ou l'autre produit ou sur un des produits de consommation tels que : la cigarette, la bière, les produits pétroliers, la communication téléphonique, l'exploitation minière dont l'implantation et la prolifération de ces dernières années à Lubumbashi n'est pas sans polluer l'environnement, etc. C'est le cas de la rivière Kafubu dont les enquêtes sont encore menées pour connaître ce responsable de pollution.

L'argent de ce fonds devrait être versé dans un compte ouvert au niveau de chaque province et un service technique pourrait être crée pour en décider sur l'affectation. Il pourrait être composé des experts de différentes divisions provinciales. Concrètement le comité de gestion du fonds serait composé des membres désignés par le gouverneur de province par arrêté délibéré en conseil des ministre.

\section{Conclusion}

Il ressort du développement de notre étude qu'il existe une tension entre ce qui est et ce qui doit être dans les établissements de santé de Lubumbashi et particulièrement à la maternité de l'hôpital général Jason Sendwe. La tension précitée consiste à la séquestration des mamans insolvables et leurs enfants à la maternité de hôpital sus-évoqué.

La pratique de la séquestration des mamans insolvables et leurs enfants dans la maternité de l'hôpital général Jason Sendwe ressemble à l'institution Romaine, la manus iniectio. A une seule différence est que, ces dernières ne sont pas enchainées. Il s'agit d'une pratique prohibée, qui énerve les principes de Droit moderne. La pratique précitée, réifie la personne humaine qui, comme le gage garantit l'exécution de l'obligation. Ce qui est inconcevable pour l'humanité et les établissements de santé d'admettre et de tolérer que les mamans insolvable et leurs enfants puisse aliéner sa personne. Aussi, il y a lieu de conclure que, l'hôpital général Jason Sendwe se rend lui-même justice à la place des juridictions compétente habilitée à trancher ce genre de litige. 
De manière générale, la pratique de la séquestration des mamans insolvables et leurs enfants à la maternité de l'hôpital général Jason Sendwe est encouragé par les prescrits de la conférence de Bamako de 1989 qui nous renseigne que "pas d'argent pas de soins de santé de qualité », chaque Etat doit subventionner dans les limites de ses moyens ces établissements de santé. Ces derniers doivent s'autofinancer à travers l'argent payé par les patients qu'ils soignent. Sur ce fait, aucun établissement de santé de premier échelon ne trouve l'intérêt de référer si tôt les patients vers le deuxième échelon. Parce qu'ils doivent les garder longtemps que possible pour s'autofinancer. Une foi référer, soit les patients meurent en cours de route "les portés mort » soit arrivaient au deuxième échelon dépouiller par le premier échelon et ils n'ont rien pour payer le prix du jour. La conséquence est la séquestration des patients insolvables jusqu'au paiement complet de leur dette.

\section{Textes légaux}

Constitution de la République Démocratique du Congo du 18 février 2006.

Loi du 20 juillet 2006, portant Code pénal congolais.

Loi n ${ }^{\circ}$ 087/ 010 du $1^{\text {er }}$ Août 1987, Code de la famille congolais.

Loi n ${ }^{\circ} 73 / 021$ du 20 juillet 19973 portant régime général des biens, régime foncier et immobilier et régime de sûreté.

Code déontologie médicale congolais du 30 avril 19970.

Code civil congolais livre III, du 20 mai 1898.

Circulaire du 20 juin 1982, relative à la fixation de tarif dans les établissements de santé de la République Démocratique du Congo. 